\title{
Molecular Dynamics Study of Vibrational Excitation Dynamics and Desorption in Solid $\mathbf{O}_{2}$
}

\author{
Lukasz Dutkiewicz, ${ }^{*}, \dagger$ Robert E. Johnson, ${ }^{\ddagger}$ Akos Vertes, ${ }^{\S}$ and Roman Pędrys ${ }^{\dagger}$ \\ Institute of Physics, Jagiellonian University, PL 30-059 Kraków, Poland, Engineering Physics, \\ University of Virginia, Charlottesville, Virginia 22903, and Department of Chemistry, \\ The George Washington University, Washington, D.C. 20052
}

Received: September 8, 1998

\begin{abstract}
Molecular dynamics calculations were performed to describe vibrational to translational energy transfer processes leading to desorption in a low cohesive energy solid excited by a laser pulse. In this study solid oxygen crystals were vibrationally excited and the redistribution of energy in the solid was followed for several nanoseconds. In a closed system, representing bulk processes, the energy transfer to the lattice occurred slowly at first while the vibrational modes equilibrated rapidly. This was followed by a short period of very rapid energy transfer to the lattice and full equilibration. The anharmonic nature of the $\mathrm{O}_{2}$ potential, the density of excitation, and the lattice structure were identified as the factors determining the rate of internal vibration to lattice energy transfer. In addition, melting, leading to molecular diffusion, was shown to lead to very rapid (catastrophic) equilibration in the closed system. The addition of an interface with the vacuum allowed desorption/ablation. Such a sample could expand, dramatically slowing the equilibration process. The vibrational energy excitation rate, which changes with laser pulse length, was shown to affect both the conversion from the internal vibrational to lattice heating and the desorption efficiency. The competition between energy transfer to the lattice and material expansion was such that desorption is a thermal process at low excitation densities corresponding to low energy transfer rates. Ablation occurs at high excitation densities for which the vibrational to translational energy transfer rates produce a pressure pulse causing rapid expansion. The ejections in the two regimes were shown to exhibit distinctly different properties. At high excitation density the ejection in the early stage is dominated by forward directed, "hot" clusters that evaporate, so the ablation process may be thought of as forming a nanospray.
\end{abstract}

\section{Introduction}

The discovery that large organic molecules can be carried into the gas phase as whole molecules following laser excitation of matrix materials is an important new tool for the mass spectrometry of biomolecules. ${ }^{1,2}$ In this process, often referred to as MALDI (matrix-assisted laser desorption and ionization), the matrix materials are volatile and typically composed of small organic molecules. These materials are often excited by a UV laser pulse, but recently IR lasers have been used. ${ }^{3,4}$ Whereas many aspects of MALDI are roughly understood, ${ }^{5-9}$ there is no model for describing the relationship between the internal energy conversion processes and the desorption and ionization processes. Obtaining a model of the desorption process could help to better understand laser-induced desorption (LID) and, possibly, MALDI. An aspect that is only qualitatively understood in LID is the conversion of internal energy to lattice motion and desorption. Therefore, we use molecular dynamics calculations (MD) to simulate the desorption from a vibrationally excited, low cohesive energy, molecular solid. Although typical matrix materials have a number of internal degrees of freedom, we have chosen to study a simple system. The potential relevance of these results to MALDI is discussed at the end of the paper.

Here, solid oxygen is used as a model material to study the effect of excitation by a laser pulse on a weakly bound molecular

\footnotetext{
† Jagiellonian University. E-mail: ldutkiew@if.uj.edu.pl, ufpedrys@ cyf-kr.edu.pl.

University of Virginia. E-mail: rej@virginia.edu.

$\S$ The George Washington University. E-mail: vertes@gwu.edu.
}

solid. Oxygen was chosen since it has one internal degree of freedom that couples poorly to the lattice vibrations. This allows us to examine the evolution of the deposited energy and the loss of surface in a model system via MD simulations. In earlier MD simulations of LID $^{10}$ and MALDI, ${ }^{11-16}$ the internal vibrations were either not represented or represented in an approximate way. The studies here are roughly related to those of Zhigilei et al. ${ }^{12,13}$ in which "breathing sphere" molecules are used. Vertes et al. ${ }^{14-16}$ described desorption of a fully modeled organic molecule from a small sample. There are also numerous calculations for desorption of individual molecules adsorbed on a surface, and vibrational to translational $(\mathrm{V}-\mathrm{T})$ energy transfer between individual molecules is treated in standard texts. Although models for $\mathrm{V}-\mathrm{T}$ transfer in a highly excited lattice exist, ${ }^{7,17,18}$ these are not well tested, nor are the models that have been proposed for desorption. ${ }^{9}$ Therefore, we consider large samples and long times (up to a few nanoseconds) using a "matrix" composed of a simple diatomic molecule. This allows us to excite an internal degree of freedom and to track the energy flow. That is, the target molecules are vibrationally excited in order to represent the laser-induced excitation of the material.

The primary objective of this study is a description of the energy flow from the vibrational modes into lattice motion. In addition, the change in the nature of this flow in the presence of a vacuum interface and in a matrix containing imbedded guest molecules is studied. We present results for desorption of both the matrix and the guest molecules. To accomplish these goals, molecular dynamics calculations for a closed periodic system were carried out first. In this way, the influence of the boundaries 
on the results of the calculation are minimized. Instantaneous as well as a few hundred picoseconds long excitation pulses were examined, and the time evolution of the system was followed for up to $3 \mathrm{~ns}$. In order to represent the desorption process, the boundary conditions were changed to allow molecules to exit from a free surface, resulting in molecule by molecule desorption at low excitation densities and ablation at high excitation densities. Preliminary results were published recently. ${ }^{19}$

\section{The Model}

Solid oxygen is a particularly friendly target for a molecular dynamics simulation since the lack of a molecular dipole moment allows for an accurate treatment of the structure and of the cohesive energy of the solid using short range, binary, van der Waals intermolecular forces. Here Lennard-Jones (LJ) pair potentials are used to describe the interaction between the $\mathrm{O}$ atoms in neighboring molecules. The $\mathrm{LJ}$ parameters are $0.0038 \mathrm{eV}$ and $2.988 \AA^{-1}$. In addition, a Morse intramolecular interaction is used to describe the internal motion of each $\mathrm{O}$ atom in an $\mathrm{O}_{2}$ molecule. The Morse parameters are $D_{\mathrm{e}}=5.19$ $\mathrm{eV}, \alpha=2.958 \AA^{-1}$, and $r_{\mathrm{e}}=1.2 \AA^{20} .^{2}$ In earlier simulations of electronically-stimulated desorption (electronic sputtering) of low-temperature condensed-gas solids by fast ions, solid oxygen was also used as a model material. ${ }^{20,21}$. In those studies, a cylindrical region was excited to represent the excitation produced along the path of the ion and the $\mathrm{O}_{2}$ well depth was changed to allow better coupling to the lattice, hence, shorter integration times. Here the full $\mathrm{O}_{2}$ well depth was used in all but a few studies, the excitation was uniform or varied only with depth, and much larger samples and longer times were studied.

Since a principal objective of these calculations is to follow the transfer of vibrational energy into lattice motion, a fully flexible model of the oxygen molecule was used. A molecular dynamics code used earlier in the study of atomic solids ${ }^{22,23}$ was modified to include the intramolecular forces. ${ }^{24}$ In order to speed up the calculations the RESPA (Reference System Propagator Algorithm), multiple-time scale algorithm due to Tuckerman et al. ${ }^{25}$ was applied. In this method intermolecular forces related to the slow translational motion are updated less frequently than the intramolecular forces. A performance gain of a factor of 5 was achieved in comparison with a conventional integration scheme without loss of accuracy. A variable time step was also used to improve the calculation speed. ${ }^{24}$ The size of the time step is controlled by maximum displacement and maximum acceleration in each interval. This also guards against inaccuracy for high-energy encounters between oxygen molecules. The studies related to ion tracks were on amorphous samples $;{ }^{20,21}$ here crystalline samples ${ }^{26}$ were used, either hightemperature $\gamma$-oxygen equilibrated at $50 \mathrm{~K}$ or $\alpha$-oxygen equilibrated at $10 \mathrm{~K}$. When desorption is considered, the vacuum interface is in the $z$-direction. In most studies both boundaries in the $z$-direction were open; in a few calculations a substrate was used.

After a ca. 30 ps long equilibration period, a randomly chosen set of molecules was excited with a given energy put into their vibrational mode in the form of equal, outward-directed momentum impulses to the two $\mathrm{O}$ atoms. We write $[E(\mathrm{eV}) \times$ $n \%$ ] to denote the average excitation energy per molecule, with $n \%$ the percent of the target molecules in the MD sample being excited with energy $E$. Periodic boundary conditions in all directions were used in the first studies of energy flow. Subsequently, an interface with the vacuum was introduced.

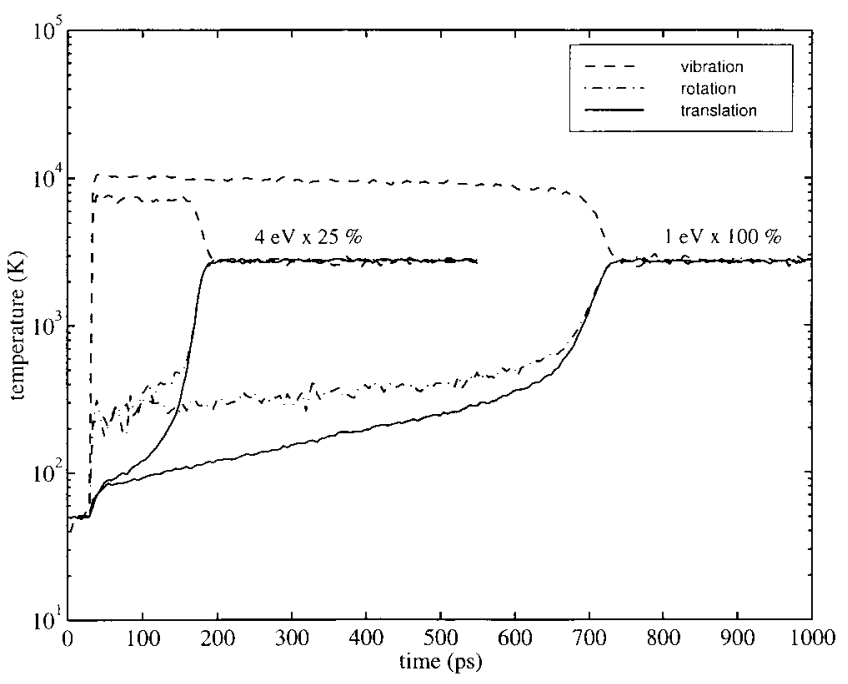

Figure 1. Comparison of the evolution of the average vibrational, translational, and rotational kinetic energy given as a temperature for two vibrationally excited samples of solid oxygen $\gamma$-crystal (512 molecules) in a periodic box. In one sample each $\mathrm{O}_{2}$ is given $1 \mathrm{eV}$ of vibrational energy; in the other every fourth $\mathrm{O}_{2}$, chosen randomly, is given $4 \mathrm{eV}$.

2.1. Lattice Heating. The time evolution of the mean kinetic energy (temperature) in the lattice motion and in the internal vibrations are shown in Figure 1 for a closed periodic system. This figure exhibits results for two simulations with the same excitation density: a $1.0 \mathrm{eV}$ vibrational excitation of all the molecules in the sample is compared with a $4.0 \mathrm{eV}$ excitation of $25 \%$ of the molecules. It is seen that the temperature in each of these modes eventually converges to the same equilibrium value for both simulations. Because the figure only shows the kinetic energy in each mode and the internal potential is anharmonic, at small times the vibrational temperatures differ between the 1 and $4 \mathrm{eV}$ cases. It is also seen that the rotational modes, in which the molecular orientation oscillates, contain more energy initially than the translational modes. However, when full equilibration is attained, all modes reach the same average energy. Full equilibration is seen to occur after a long period of very slow nearly linear growth of lattice kinetic energy, which is followed by a rapid, catastrophic heating and a nonlinear increase in lattice kinetic energy until the equilibrium temperature is reached. Below we discuss these two regions.

Whereas the qualitative features of the energy evolution for both excitations shown in Figure 1 are the same, the evolution is seen to be much faster for the lower density, higher energy excitation case. There are a number of reasons for this initially surprising result. One contributing effect is the anharmonicity of the vibrational potential, since anharmonicity increases with increasing vibrational excitation in a Morse potential. ${ }^{18}$ The importance of the anharmonic nature of the internal vibrational mode is shown by comparing the evolution of an excited oxygen sample prior to the rapid increase in heating to the excitation of artificially altered oxygen samples in Figure 2. Results for an oxygen sample using the standard Morse parameters above, which we will refer to below as "real" oxygen, are compared to results for an oxygen sample but with the intermolecular well depth reduced by $50 \%$ and with a sample having harmonic intermolecular potentials but with the same well depth as solid oxygen.

It is seen in Figure 2 that there is an immediate increase in the lattice temperature for both the "real" and harmonic $\mathrm{O}_{2}$. This is the case for all weakly coupled oscillators. The exchange of energy between oscillators, to be discussed, is through the 


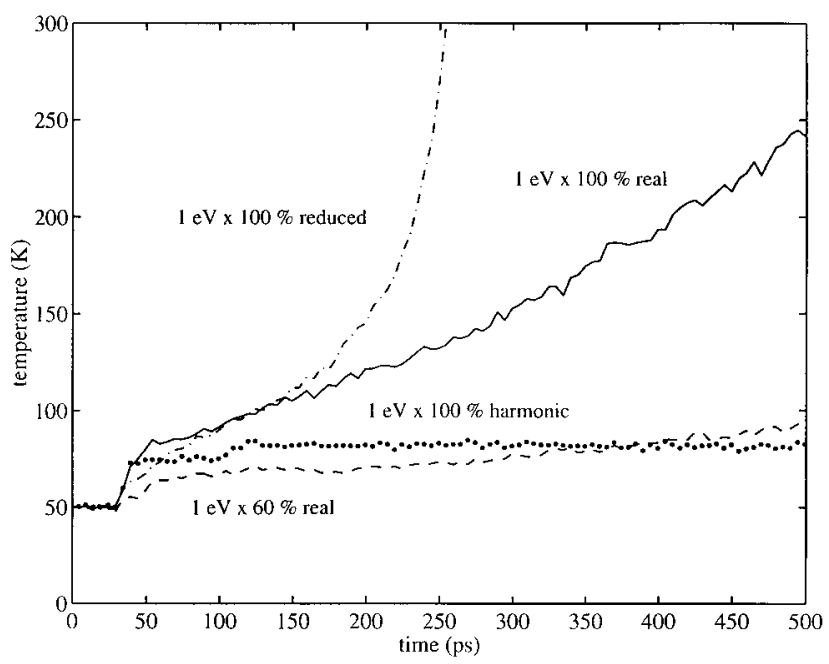

Figure 2. Comparisons of the evolution of the average translational kinetic energy, given as a temperature, in a vibrationally excited solid oxygen $\gamma$-crystal (512 molecules) in a periodic box. "Real" oxygen samples described using the Morse potential with all molecules excited (solid) and the same sample with $60 \%$ of the molecules excited (dashed) are compared with "artificial" oxygen samples: Morse potential reduced by $50 \%$, (dash-dot) and a harmonic potential replacing the Morse potential (dotted). The points are averaged over five snapshots in $2 \mathrm{ps}$ intervals.

weak lattice interaction. Therefore, the relative time that energy spends in the lattice determines the temperature jump. That is, the ratio of the mean energy in the lattice to that in internal vibrational energy is roughly equal to the ratio of the vibrational periods. After this initial heating, it is seen that the "real" $\mathrm{O}_{2}$ lattice (solid curve) slowly heats up but the harmonic $\mathrm{O}_{2}$ sample (dotted curve) essentially does not change over the time period studied. Therefore, the anharmonicity of the intramolecular potential influences the lattice heating rate. The anharmonic potential affects the energy transfer in two ways. First, with increasing excitation energy, $E$, the frequency of the Morse oscillator, $\omega_{\text {Morse }}=\alpha \sqrt{(2 / m)(D-E)}$, decreases, resulting in smaller differences in frequency between the internal and lattice modes and, hence, the possibility of more efficient energy transfer between the internal vibrations and the lattice oscillations. ${ }^{18}$ In addition, increased anharmonicity results in the expansion in the average size of an excited molecule and increasing distortion of the lattice. Treating molecular excitations by increasing the molecular size has been used in a number of simulations of LID and biomolecule desorption. ${ }^{10,12,13,27}$ Because of the importance of the anharmonic potentials, models such as the CHARMM program, which employ harmonic potentials in describing complex molecules, ${ }^{14,28}$ should be used with caution when modeling vibrationally-induced desorption.

The role of the anharmonic potentials is further shown in Figure 2 by comparing the results for samples in which only $60 \%$ of the $\mathrm{O}_{2}$ are excited by $1 \mathrm{eV}$. One such sample is "real" $\mathrm{O}_{2}$ (dashed curve) and in the other the well depth was reduced by $50 \%$ (dot-dash curve). The anharmonicity in the Morse potential $\left(\omega_{\text {Morse }}-\omega_{\text {harmonic }}\right) / \omega_{\text {Morse }}$ depends inversely on the square root of the well depth minus the excitation energy, corresponding to a much higher anharmonicity for the smaller well than that for the "real" $\mathrm{O}_{2}$. It is seen that the heating rate is dramatically increased for the reduced well depth because the frequency differences with the lattice are smaller and the mean molecular size is larger. In the simulations for fast-ioninduced desorption, well depths of less than $1 \mathrm{eV}$ were used, resulting in very rapid energy exchange with the lattice. ${ }^{21}$

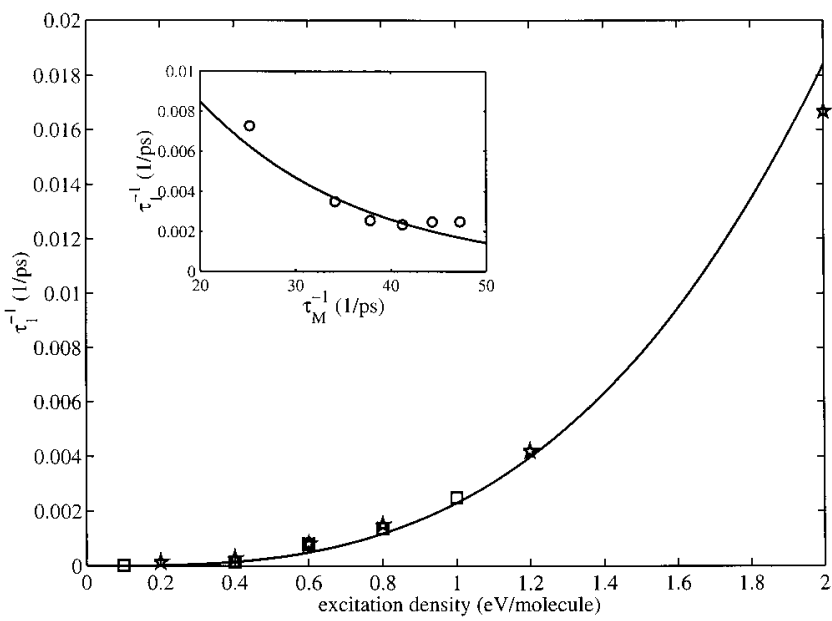

Figure 3. Rate of translational temperature growth in time in the slow growth regime, $\tau_{1}^{-1}=(\partial T / \partial t) / T_{1}$, vs excitation energy density per molecule in bulk $\gamma-\mathrm{O}_{2}$ crystal. The points are fitted with a cubic: ( $\square$ ) $1 \mathrm{eV}$ excitation energy; ( $\star 2) 2 \mathrm{eV}$ excitation energy. In the inset, dependence of $\tau_{1}^{-1}$ vs the frequency of the excited Morse oscillators, $\tau_{\mathrm{M}}{ }^{-1}$, is shown for the slow growth regime. The data points are very roughly fitted with an exponential.

In the following we extract the time constants for the $\mathrm{V}-\mathrm{T}$ energy transfer, first in the region of slow energy transfer and then in the region of very rapid transfer, which we refer to as "catastrophic" energy transfer. For these samples and excitation energy density the region of nearly linear growth of the $T$ vs $t$ graphs in Figures 1 and 2 are found to be closely fit by exponentials. Therefore, in this region a simple rate equation describes the temperature profile. We use the form $T(t)=T_{1}$ $\exp \left(t / \tau_{1}\right)$, where $T_{1}$ is the temperature at the beginning of the linear rise and $\tau_{1}$ is the time constant. Fitting curves like those in Figures 1 and 2 gives the extracted values of $\tau_{1}$ in Figure 3. The inset in Figure 3 gives the dependence of $\tau_{1}{ }^{-1}$ on the initial period of the the excited molecules, $\tau_{M}{ }^{-1}=(\alpha / 2 \pi) \times$ $\sqrt{(2 / m)(D-E)}$. It is seen that the coupling increases with increasing $\tau_{\mathrm{M}}$, consistent with models for $\mathrm{V}-\mathrm{T}$ energy transfer. ${ }^{18}$ A rough fit to the resonance function, $\exp \left(-\tau_{\mathrm{L}} / \tau_{\mathrm{M}}\right),{ }^{29,30}$ is given, where $\tau_{\mathrm{L}}$ is the time constant for the lattice. Of course, $\tau_{\mathrm{L}}$ decreases with increasing temperature, leading in part to the rapid increase seen at higher $T$. Here we obtain $\tau_{\mathrm{L}}$ from the fit and note that it is close to $(v / 2 \sigma)$, where $\sigma$ is the LJ parameter and $v$ is the mean velocity determined from the lattice temperature ( $v$ is of the order of $1 \AA / \mathrm{ps}$ and the fit value of $\tau_{\mathrm{L}}$ $=0.06 \mathrm{ps})$.

A second factor affecting the lattice heating rate in the slow heating regime is the energy density of excitation. This is clearly seen by comparing the dashed curve in Figure 2, in which $60 \%$ of the molecules within the sample were excited, to the case for full excitation (solid). A number of calculations have been carried out for various excitation densities with fixed excitation energy (i.e., fixed anharmonicity in individual oscillators). For 1 and $2 \mathrm{eV}$ excitations, the time constant, $\tau_{1}{ }^{-1}=(\partial T / \partial t) / T_{1}$ for the slow nearly linear rise in the lattice temperature seen in Figures 1 and 2, is plotted vs energy density in Figure 3. At the lowest possible excitation density, one excited $\mathrm{O}_{2}$ in a sample of cold molecules, the loss of vibrational energy is negligible over the time scales studied. Therefore, it is the exchange of energy among the excited oscillators $\left(\mathrm{V}-\mathrm{V}^{\prime}\right.$ energy transfer) that produces lattice heating in this solid. Such a process cannot be represented using "breathing spheres" to represent molecules. ${ }^{12}$ The heating rate is also seen to depend nonllinearly on the density of excited molecules, as can be seen in Figure 3. 
That is, $\tau_{1}^{-1}$, is not determined just by the vibrational frequency of the lattice, as it would be for an excited oscillator surrounded by cold molecules; rather it depends on the density of the excited oscillators for any given excitation energy. Writing $\tau_{1}{ }^{-1} \sim$ $\tau_{\mathrm{L}}{ }^{-1} f\left(n_{\mathrm{ex}} / n\right)$, where $\left(n_{\mathrm{ex}} / n\right)$ is the fraction of the oscillators excited to the initial frequency, the line drawn through the data in Figure 3 is cubic in $\left(n_{\mathrm{ex}} / n\right)$.

The region of very rapid, catastrophic lattice heating can also be approximated by the form $T(t)=T_{2} \exp \left(t / \tau_{2}\right)$, where $T_{2}$ is the onset of the exponential dependence. The heating rate, given by the fitting parameter $\tau_{2}{ }^{-1}$, is again found to be determined by $\left(\tau_{\mathrm{L}} / \tau_{\mathrm{M}}\right)$, but the prefactor $T_{2}$ is 1 order of magnitude larger than $T_{1}$. We also note that the lattice heating rate in this regime is close to the vibrational cooling rate found for gas-phase $\mathrm{O}_{2}$ when scaled by the pressure. That is, when comparing laboratory data on vibrational cooling, one typically plots $(P \tau)$ vs $T^{-1 / 3}$ where $P$ is the gas pressure and $\tau$ is the time constant extracted from vibrational cooling data. ${ }^{31}$ Calculating the pressure in the MD simulations, we find for ca. $300 \mathrm{~K}, P \tau_{2}=2 \times 10^{3} \mathrm{GPa} \mathrm{ns}$, which is roughly comparable to the gas phase data.

Comparing the two regimes, we note that the onset temperatures, $T_{1}$, in the slow heating regime are all similar. However, the onset of the rapid, catastrophic heating regime, $T_{2}$, is not only much larger than $T_{1}$ but is found to increase with increasing excitation density at a fixed excitation energy. This trend can roughly be understood in terms of the phase diagram for $\mathrm{O}_{2}$. That is, below we show that the rapid heating regime follows the formation of a melt, and the $\mathrm{O}_{2}$ phase diagram indicates that with increasing pressure the transition to a melt occurs at increasing temperature. Calculating the internal pressure in our simulations and noting when a melt forms, we find the same trend here. Therefore, the trend for $T_{2}$ described above is consistent with the onset of rapid heating occurring in the formation of a melt. Since the pressure and lattice potential energy both follow the temperature, none of these parameters alone is an indicator of the catastrophic heating onset.

2.2. Vibrational Equilibration. Whereas the lattice and vibrational modes were seen to equilibrate slowly at first (Figure 1), the vibrational energy exchange between the individual oscillators is found to be remarkably efficient if a significant density of oscillators is excited $\left(\mathrm{V}-\mathrm{V}^{\prime}\right.$ transfer). Because of this, the distribution of the vibrational states in the sample evolves toward a Boltzmann-like distribution of vibrational energies much faster than the convergence between the temperatures in different modes takes place. This is seen in Figure 4 for $1.0 \mathrm{eV} \times 100 \%, \gamma-\mathrm{O}_{2}$ system. In this figure the distribution of vibrational energy in the sample given by $P(E)$ is displayed vs time. The initial distribution at $30 \mathrm{ps}$, which is not shown, is almost a $\delta$ function. This distribution rapidly broadens due to the coupling between the oscillators. It eventually approaches a Boltzmann distribution, $P(E) \sim \exp \left(-E / k T_{\text {vib }}\right)$ at ca. $600 \mathrm{ps,}$ where $T_{\text {vib }}$ is a vibrational temperature at this time. This temperature of course varies slowly with time, as seen in Figure 1 , due to energy transfer to the lattice. This behavior is much more obvious for the sample of harmonic oscillators studied in Figure 2. Over the times studied there is almost no energy loss to the lattice by the harmonic oscillators after the initial temperature jump, so that the internal vibrations equilibrate separately. Because the energy loss from the $1 \mathrm{eV}$ excited Morse oscillators is initially slow, from Figure 4 we find that the Morse oscillators approach a Maxwellian at early times. This behavior was found to be general in the samples studied.

The broadening of the peak in the vibrational distribution produces both higher and lower energy oscillators at a rate

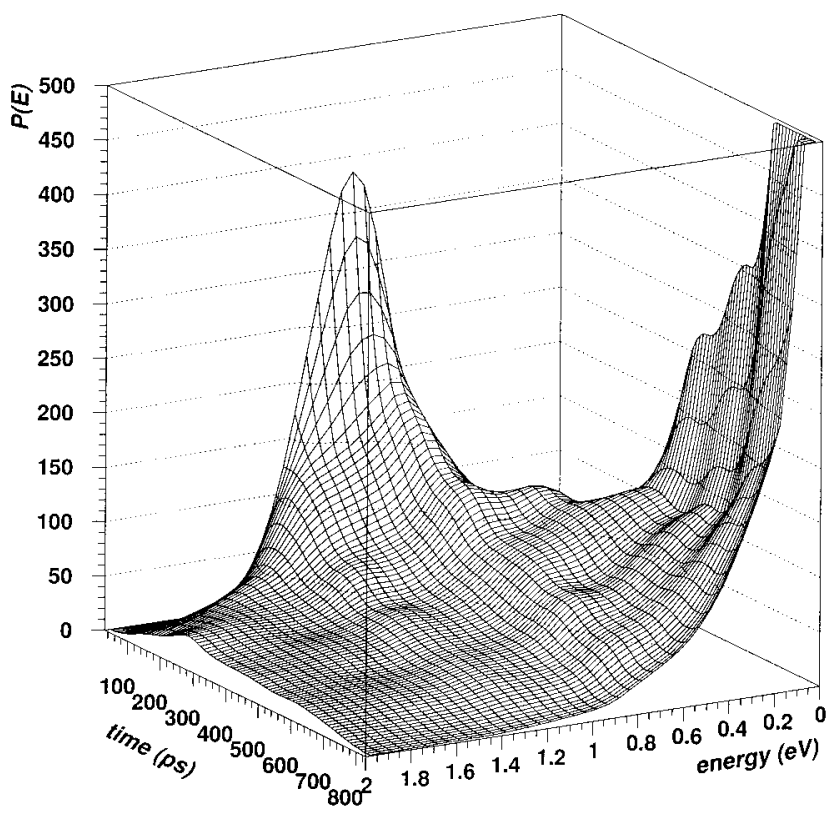

Figure 4. Evolution of vibrational energy distribution, plotted for a solid oxygen $\gamma$-crystal (512 molecules) excited uniformly with $1 \mathrm{eV}$ per molecule. The probability, $P(E)$ of any molecule having a total (kinetic + potential) vibrational energy has been plotted. The distribution has been spline smoothed in order to expose the qualitative features of the distribution.

determined by the energy exchange. This in turn is determined by the lattice period. ${ }^{29}$ The amount of the exchange is also determined by the coupling to the lattice. In each exchange between anharmonic oscillators, there is a small mismatch of the energies that is left in the lattice as heat. Since the frequencies of anharmonic oscillators decrease with increasing excitation energy, the expansion and lattice distortion increase. Therefore, the coupling to the lattice also increases somewhat, as discussed above. Consistent with this, it is seen in Figure 4 that the higher energy modes are quenched faster. As time increases and an increasing amount of energy has been transferred to the lattice, the vibrational distribution gradually shifts to lower energies. Although the lattice energy gradually increases, this effect alone does not appear to significantly improve the coupling until structural changes occur that produce the very rapid, catastrophic heating.

It is found by monitoring individual oscillators that the broadening of the vibrational distribution occurs first by exchange between the excited oscillators, rather than by transfer of excitation to unexcited $\mathrm{O}_{2}$ even if the excited oscillators are not nearest neighbors. Therefore, the proximity of the excited $\mathrm{O}_{2}$ affects the rate of energy exchange among the $\mathrm{O}_{2}$ oscillators. Whereas after the initial temperature jump the harmonic oscillators exchange vibrational energy without heating the lattice, it is this exchange among anharmonic oscillators that leaves residual heat in the lattice, causing the slow temperature rise seen in Figures 1 and 3. Since that exchange depends on the presence of neighboring oscillators, $\tau_{1}{ }^{-1}$ was found to depend nonlinearly on the density of excited molecules, as discussed, and a roughly quadratic dependence on the excited oscillator density was found for $\tau_{2}{ }^{-1}$.

The heating of the lattice eventually leads to structural changes, melting, and decomposition. This is best seen for an $\alpha-\mathrm{O}_{2}$ sample, since the structure of $\gamma-\mathrm{O}_{2}$ is much more disordered, having a pair correlation function resembling that of the liquid phase. ${ }^{26}$ In Figure $5 a-3 d$ the evolution of the structure of the $1.0 \mathrm{eV} \times 100 \% \alpha-\mathrm{O}_{2}$ crystal is shown, and in 

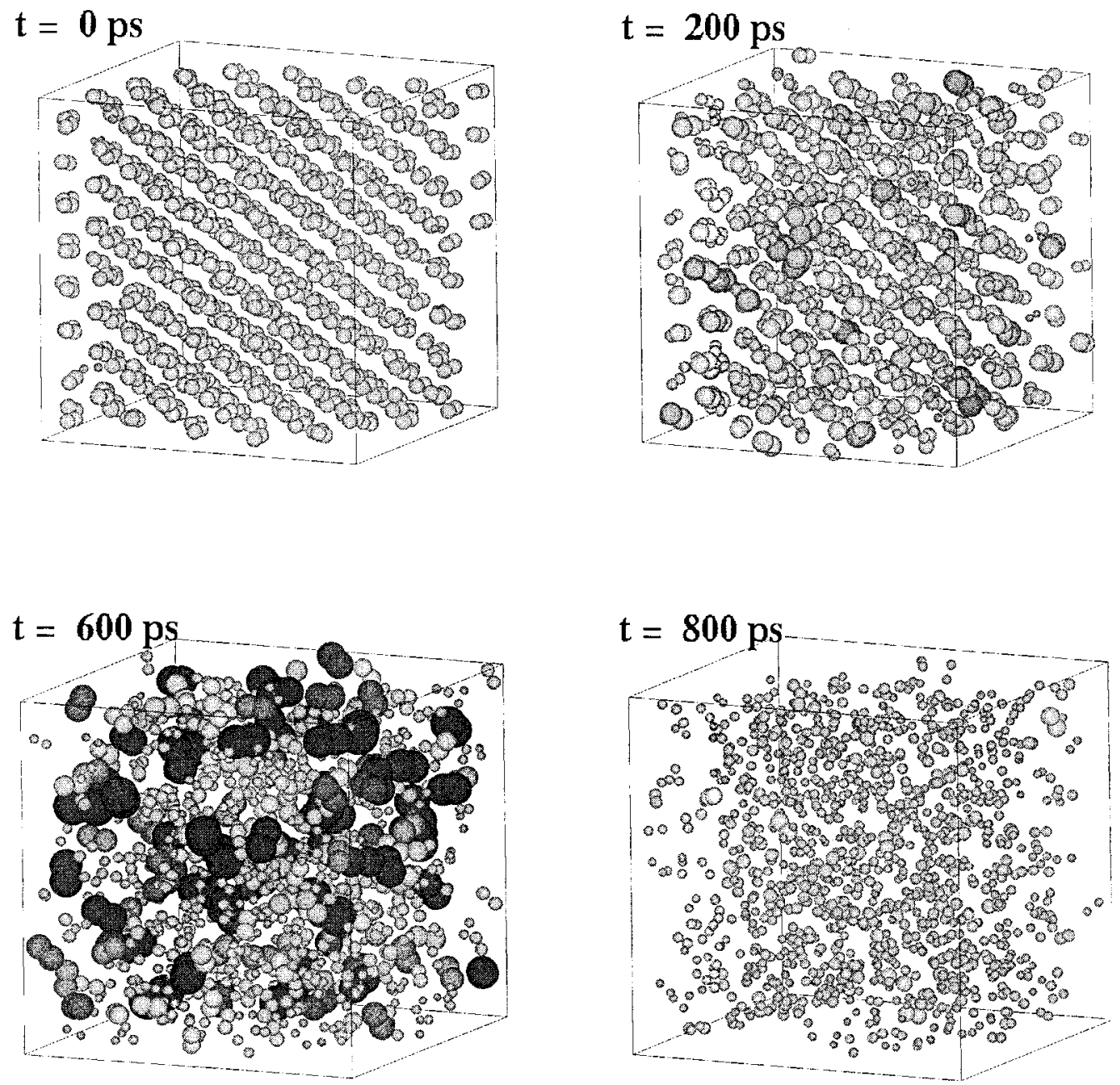

Figure 5. A 3D visualization of a bulk $\alpha-\mathrm{O}_{2}$ crystal sample (550 molecules) at times $t=0,200,600$, and 800 ps after vibrational excitation (1 $\mathrm{eV} \times 100 \%$ ). The atoms are represented as spheres with radius and brightness proportional to their total (potential + kinetic) vibrational energy. At $200 \mathrm{ps}$ the material is in the linear energy transfer regime, whereas at $600 \mathrm{ps}$ it approaches the rapid energy transfer regime. At $800 \mathrm{ps,} \mathrm{the}$ vibrational energy is transferred to translational and rotational modes and the sample equilibrates at ca. $3000 \mathrm{~K}$.

Figure 6a,b the lattice kinetic energy and the pair correlation function are shown. It is seen from the lattice kinetic energy plot for the $\alpha-\mathrm{O}_{2}$ sample that a phase change is suggested by the abrupt increase in temperature at 200 ps. This is consistent with both the change in structure seen in Figure $5 \mathrm{~b}$ and in the correlation (Figure 6b), which suggests a liquid-like phase. Similarly, between 600 and 800 ps the catastrophic lattice heating occurs and the solid becomes gas-like, resembling spinodal decomposition ${ }^{11}$ (Figure $5 \mathrm{~d}$ ). This exhibits a relatively flat pair correlation function (Figure 6b) and the potential energy becomes positive.

The above changes are also accompanied by changes in the mean square displacement of the molecule. ${ }^{19}$ The rapid, nonlinear temperature increase described earlier is found to occur close to those times at which a structural transition occur, allowing the molecules to migrate. That is, a high level disorder associated with a phase change appears to be required for rapid equilibration. This allows the excited oscillators to diffuse, collide, and exchange vibrational energy, which increases the transfer of energy to the lattice, as discussed above. Therefore, although the classical version of a "phonon avalanche" is observed here, the mechanism is very different from that described by Fain and Lin. ${ }^{18} \mathrm{We}$ find that it is the collapse of the lattice structure that allows the more rapid heating by allowing more efficient interaction of the excited oscillators.
2.3. Pulse Length. In order to model the effect produced by the typical laser pulse, the excitation process should not be instantaneous but should be extended over a period of time. Typical experimental pulse lengths are technically beyond the reach of our present computing capabilities but the 500 and 1000 ps long pulses used in the present calculation are both relevant to some laser excitation experiments and can give guidance when longer pulses are of interest.

The qualitative behavior of the system does not change from the description above when long pulses are used instead of $\delta$ pulses. That is, although there is no initial temperature jump the temperature evolution of the long-pulse excited systems presented in Figure 7 also shows a slow rise followed by a rapid energy transfer to the lattice (not shown) leading to equilibration in the various modes. However, the continuously growing excitation density during the pulse results in a steepening slope in the temperature plot. It is interesting to note that the samples absorbing a long pulse reach equilibrium faster than samples heated by a $\delta$ function pulse with the same total energy (1.0 $\mathrm{eV} \times 60 \% \delta$ vs 500 ps pulse). The sample was excited with the 500 ps long pulse equilibrated after ca. $1 \mathrm{~ns}$. The vibrational excitations and the redistribution of the vibrational energy cause lattice disorder. Therefore, exciting a new oscillator in a partially disordered lattice increases the efficiency for energy transfer to the lattice. 

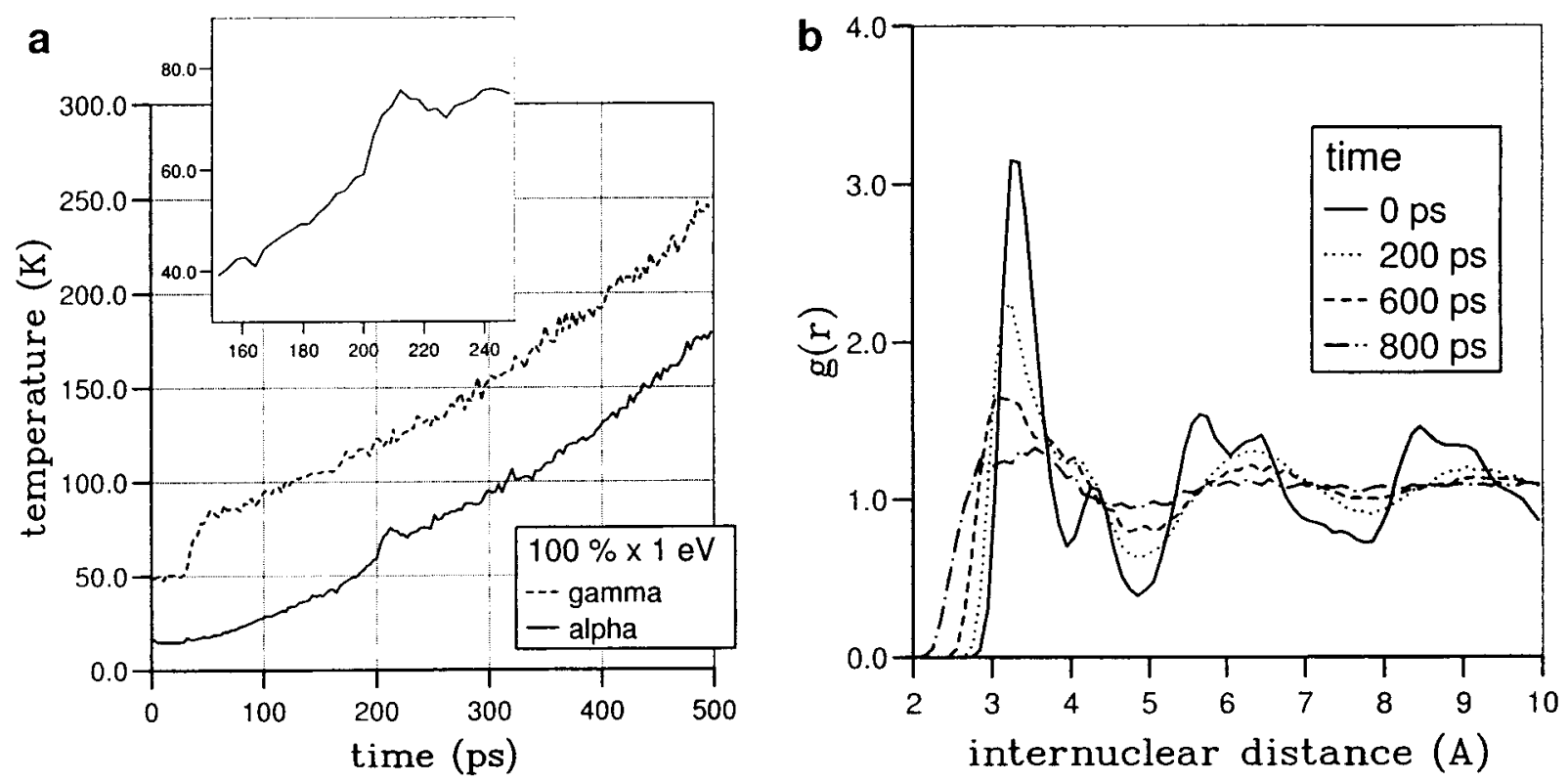

Figure 6. (a) Translational temperature evolution in a vibrationally excited solid $\alpha$ - and $\gamma$-oxygen in a periodic box. The points are averaged over five snapshots in 2 ps intervals. Inset: detail for $\alpha$-oxygen. (b) Pair correlation function in the $\alpha$-oxygen crystal plotted at critical stages of the time evolution.

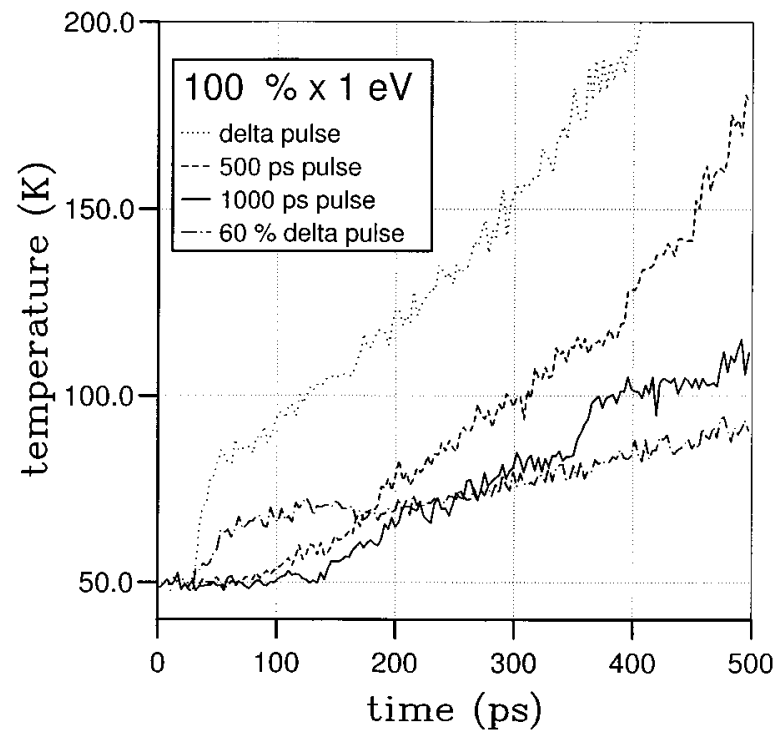

Figure 7. Translational temperature evolution of solid oxygen in a periodic box. The sample is excited instantaneously $(\delta$ pulse): (dotted) full excitation; (dash-dot) $60 \%$ of molecules excited. Comparison to excitation pulses uniformly spread over a certain time: all molecules are to be excited within $0.5 \mathrm{~ns}$ (dashed) and $1 \mathrm{~ns}$ (solid). The points are averaged over three snapshots in 2 ps intervals.

2.4. Impurities and Analytes. The introduction of impurities into the matrix affects the rate at which vibrational energy is transferred to the lattice. ${ }^{19}$ As a first step we created an alloy of $50 \%$ normal $\mathrm{O}_{2}$ molecules that were vibrationally excited and $50 \% \mathrm{O}_{2}$ with altered mass that were not excited. The effect of increased mass on the vibrational modes is to decrease the frequencies and, for a Morse potential, increase the anharmonicity. We found that the sample in which the altered $\mathrm{O}_{2}$ had a larger mass heated faster, whereas the reverse was true for the samples in which the mass was reduced, consistent with the discussion here on energy transfer.

In addition, a spherically symmetric large molecule of fixed radius was added, representing an analyte. The analyte and $\mathrm{O}_{2}$ interaction was modeled with a shifted Lennard-Jones potential. ${ }^{27}$ After removing the overlapping $\mathrm{O}_{2}$ molecules, adding the large molecule, and allowing the sample to relax, the $\mathrm{O}_{2}$ matrix was vibrationally excited. An increase in the lattice heating rate by $20-30 \%$ occurred due to the disorder produced when the analytes were introduced in the bulk of the crystal. ${ }^{19}$

2.5. Desorption and Ablation. When the periodic boundaries in one direction are removed, the loss of surface material, referred to as desorption or ablation, can be modeled. In addition to allowing the loss of material, the presence of a free surface allows the target to give up the deposited energy by surface relaxation and by cooling due to the ejection of "hot" molecules. Both effects are observed in the MD studies of open oxygen surfaces. Two problems are encountered when trying to represent conditions typical to a MALDI experiment. First, for typical absorbances ${ }^{1,2}$ the excitation density deposited is nearly flat over our cell size. This size is determined, of course, by reasonable limits on the run times. Second, when a sample that is weakly attached to a substrate is uniformly excited, it typically breaks away from the substrate due to the momentum associated with the expansion of the solid. Therefore, a number of spatial excitation distributions were studied and different lower surfaces were tried in order to find operating parameters for which the physics of the desorption process is reasonably represented. It was found that any loss of translational symmetry in the crystal properties promotes breaking the sample in the place of discontinuity. Therefore, free bottom boundary has been found to be the most useful, although not perfect solution.

In our previous paper ${ }^{19}$ we compared the lattice heating rate for two samples, each excited with $2 \mathrm{eV}$ per molecule, one having the periodic boundaries in one direction removed and the other not. Whereas the latter sample behaved as described in detail in the discussion above, slow heating followed by catastrophic heating and equilibration at $6000 \mathrm{~K}$, the one with open surfaces did not heat above ca. $100 \mathrm{~K}$ in the time period studied. That is, it did not go through the catastrophic heating process. This was the case as the lattice energy went into the expansion of the solid, which in turn reduced the vibrational relaxation rate. That is, the solid expanded continuously for several hundreds of picoseconds, and consequently the density of the solid was reduced by more than $30 \%$, causing the coupling to the lattice to decrease. Therefore, the vibrational cooling to 

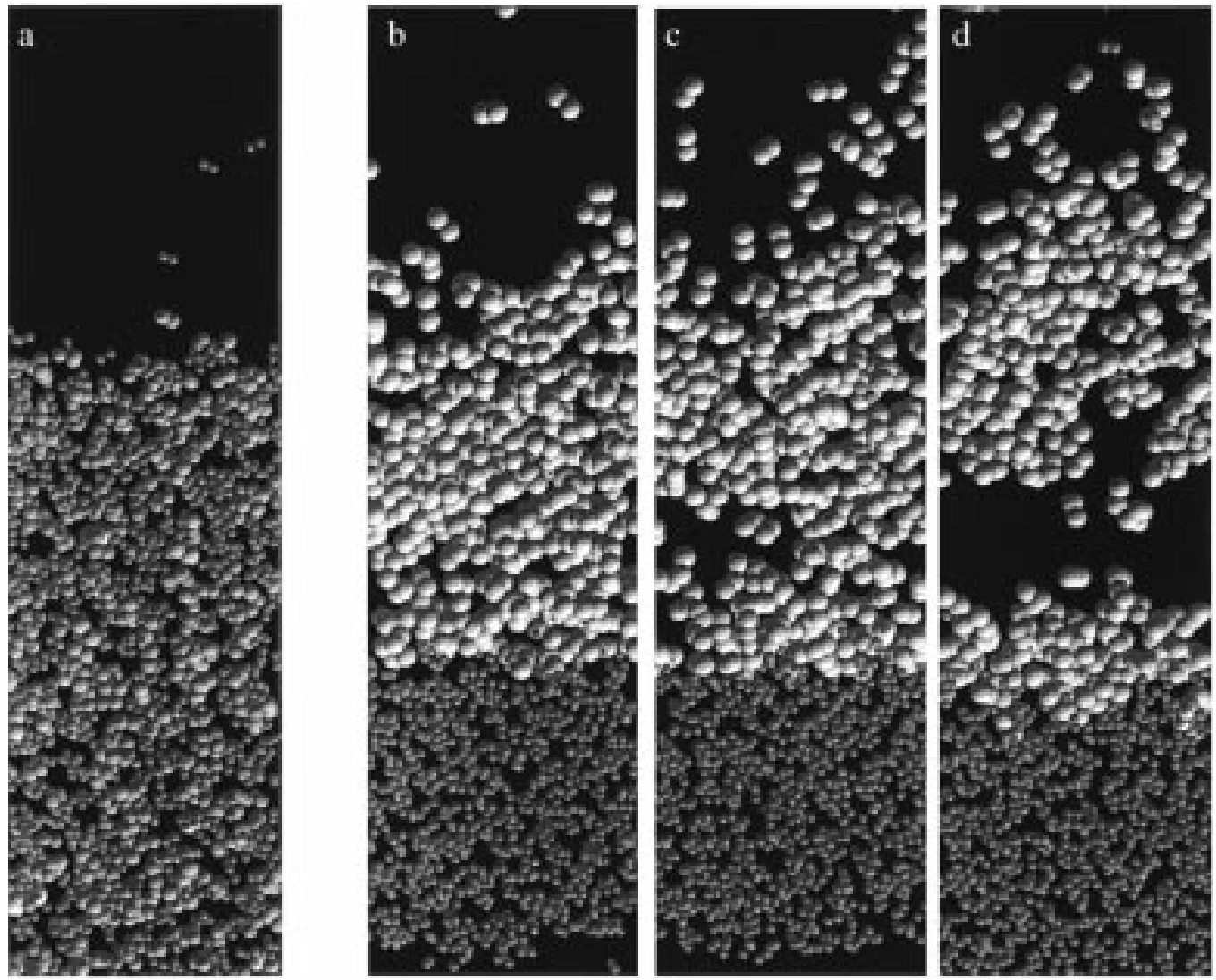

Figure 8. (a) Side view of the surface of a $\gamma-\mathrm{O}_{2}$ crystal sample uniformly excited with $1 \mathrm{eV}$, seen at $t=500 \mathrm{ps}$ after excitation. (b) $-(\mathrm{d}) \gamma-\mathrm{O}_{2}$ sample: upper half excited uniformly with $4 \mathrm{eV}$, seen at $t=5 \mathrm{ps}(\mathrm{b}), t=10 \mathrm{ps}(\mathrm{c}), t=30 \mathrm{ps}(\mathrm{d}) . \mathrm{O}_{2}$ The atoms are represented as spheres with radius and brightness proportional to their total (potential + kinetic) vibrational energy.

the lattice remained very slow. In such a sample, desorption occurred as a molecule by molecule sublimation process, as shown in Figure 8a. Although this also contributed to the cooling, the lattice expansion was the dominant effect over the run time. Consistent with an evaporative process, the desorbed molecules were found to have a kinetic temperature about $2 k T$ where $T$ is the lattice temperature. Their velocities close to the surface also exhibited a cosine distribution, and the evaporation rate was close to that for $\mathrm{O}_{2}$ at the lattice temperature.

In section 2.1 we showed that the heating rate increased with increasing excitation density and with increasing excitation energy for these Morse oscillators. In order to study possible "catastrophic" desorption, the heating rate has to exceed the rate at which the energy density is diluted by the expansion. Since the time scale for initiating the surface expansion is tens of picoseconds, then, as is seen in Figure 1, one must use a $\delta$ function pulse that has a very high excitation density. (Because of computation time we did not use long pulses, although this would allow the use of excitation densities lower than those used below.) In fact, we have shown that catastrophic heating requires that the sample must essentially melt or achieve a high level of disorder so that diffusion of excited species occurs. This "melting" is also seen in the study below. It is interesting that Beavis and co-workers have recently found that the melting is critical to protonating the analyte in MALDI. ${ }^{32}$

To handle the substrate problem and to roughly describe nonuniform excitation, a sample of 2000 molecules was constructed. Half of this sample was excited with $4 \mathrm{eV} \times 100 \%$, which for the closed sample led to catastrophic heating in less than 100 ps. The results are displayed at $3 \times$ in Figure $8 b-d$, and the ablated volume is clearly seen in the last frame. Although ablation clearly occurs, catastrophic heating is not seen when the lattice temperature is monitored. Two phases can be distinguished in the ablation process: first, the pressure in the excited part of the sample increases rapidly, causing the excited part of the sample to rapidly move outward with a proportion of the sample detaching. The detachment occurs just above the limit of the excited zone for the simple excitation geometry used. Subsequently, the flux drops by 1 order of magnitude and the process becomes evaporative. This picture of the ablation process is qualitatively the same as that found by Zhigilei et al. using breathing spheres to represent vibrationally excited molecules ${ }^{12,13}$ and is described roughly by the analytic, pressure pulse model. ${ }^{9}$

The two phases of the ablation process described above are seen in Figure 9, in which results for a high excitation density run are compared to those for a low excitation density run, as in Figure 8. For the high excitation density case, it is explicitly seen that there is a large prompt yield followed by a slower increase. The slower increase resembles that for the lower excitation density simulation in the same figure. Although these calculations cannot be run for long enough times to finalize the ejections, we clearly see in the simulations that, in addition to the molecules that escape, molecules leave the ablated volume and return to the sample. On the basis of the result in Figure 9, we estimate the yield for the prompt ejections for the high excitation density case to be about $10^{16}$ molecules $/ \mathrm{cm}^{2}$, or about 10 monolayers at a fluence of $3 \mathrm{~mJ} / \mathrm{cm}^{2}$. It should be noted, however, when comparing this yield to that obtained from experiment, that the whole energy was concentrated close (within $100 \AA$ ) to the surface and the binding energy of solid oxygen is lower than that of typical MALDI matrices.

The difference in the desorption processes in the two regimes discussed above is reflected in the state of the ejecta, both 

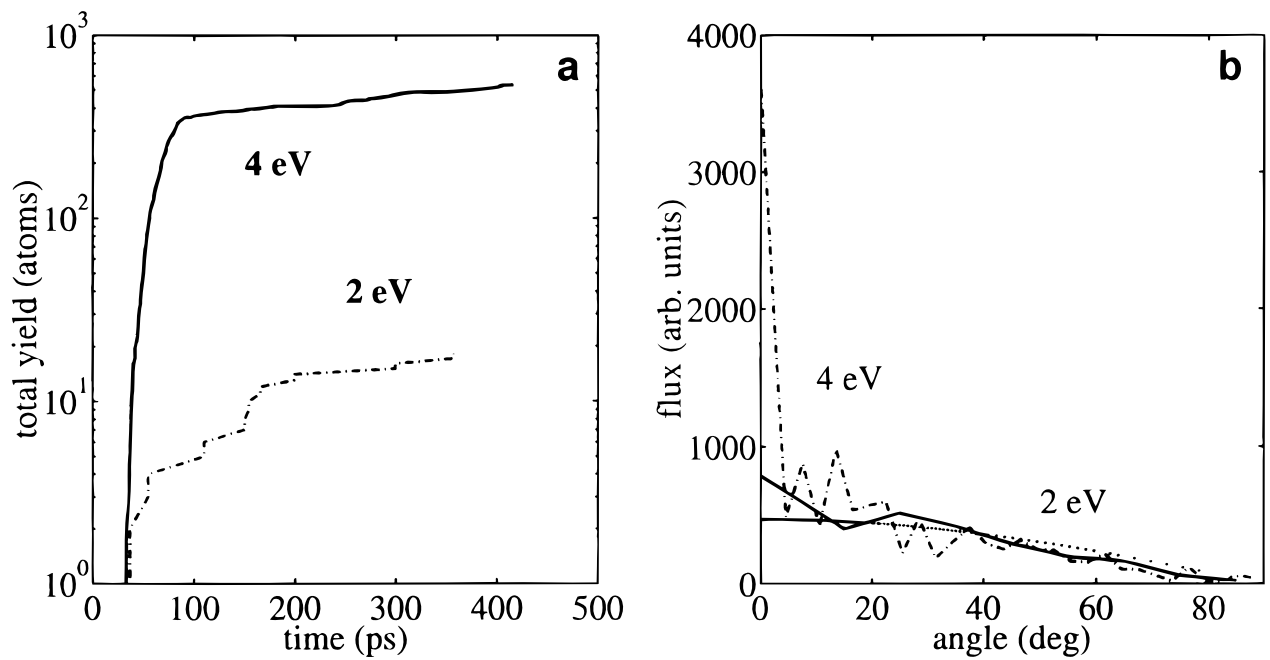

Figure 9. (a) Yield vs time for a $\gamma-\mathrm{O}_{2}$ crystal with all molecules in the surface region excited with either 2 or $4 \mathrm{eV}$. (b) Angular distributions of desorbed molecules calculated for the same samples. The distributions are normalized at large angles. The cosine distribution is given as reference by the dotted line.

angular distribution and the distribution of clusters. It is seen in Figure $9 \mathrm{~b}$ that the prompt, ablated volume is very forward directed with the later ejections exhibiting a cosine distribution. The angular distribution at low excitation densities is also cosine-like, as mentioned above. The forward directedness seen in the prompt ejections is striking. This result is in contrast to much of the discussion in the literature. That is, it is often stated that the measured forward directedness of the ejections is due to collisions in the plume, as in a nozzle. Although plume effects certainly contribute, here it is shown that the angular distribution is already determined for the prompt contribution when the ejections are close to the solid state density, as suggested earlier. ${ }^{9}$

A second characteristic is that the ablated volume experiences intense cluster emission over a few tens of picoseconds (cf. Figure 8d). This is followed by late evaporation from the solid and by evaporation of molecules from the ejected clusters, decreasing their size. In this way a plume of ejected molecules is formed. However, in the outgoing flux there is still a large fraction of dimers and trimers of these neutral molecules at the end of our run. The evaporation of molecules from the ejected clusters implies that laser ablation mass spectrometry of such material may be considered a nanospray, being analogous to electrospray in which small ejected droplets containing an analyte shed the solute molecules.

During the ablation process we find that the kinetic energy is rapidly redistributed within the residual sample. In contrast to this, the vibrational energy redistribution is much slower. This is again due to the density decrease with expansion, and the sample probably needs several nanoseconds to equilibrate. Therefore, the ejections that are initially excited retain a large fraction of their internal energy and the prompt volume ejected experiences melting. This leads, eventually, to the dissolution of the clusters, as indicated above. This energy release can in turn energize the plume. The corresponding result for the case of biomolecules is that we would expect relatively high internal temperatures initially, which can lead to fragmentation. This is mitigated by collisional cooling of the vibrational modes during the expansion process. Such modeling suggests that the cooler analyte molecules might result from ejected clusters that contain an analyte and shed the matrix molecules.

We also added a large analyte molecule, as discussed in section 2.4 , to the open surface sample and excited it with excitation densities up to $4 \mathrm{eV} /$ molecule. The sample again expanded and sublimation occurred. In addition, the large impurity molecules, if initially placed at a depth into the sample, often migrated to the surface, where they tried to desorb. The threshold for analyte desorption was found to be about $0.6 \mathrm{eV} /$ matrix molecule, for analyte mass 100 amu (see also ref 19). The migration of the analyte seen suggests that for subablation pulses segregation of the analyte occurs. Therefore, when one works close to the threshold fluence for MALDI, depletion of the analyte may result at depths below the ablated volume. This can in part be responsible for the loss of analyte signal after a number of pulses on a sample.

\section{Conclusions}

A study of energy flow in a vibrationally excited molecular solid was carried out to help understand the flow of energy in the laser-induced desorption. Low-temperature solid oxygen was used as the simplest model system. Samples of $\mathrm{O}_{2}$ were vibrationally excited, varying the excitation energy and the excitation density. The transfer of the initial vibrational energy into the lattice rotational and translational modes was studied. The calculations were carried out using closed and open samples. In the latter, desorption occurred. It was shown that, even at low excitation densities, energy exchange between the ensemble of excited oscillators, leading to a nearly Maxwellian distribution of vibrational energies, preceded the equilibration with lattice modes. Full equilibration for the closed sample occurred in nanosecond time scales, down to about 100 ps for the largest excitation densities studied.

In closed systems two distinct regimes were found for the translational temperature evolution. A slow, nearly linear heating regime and a nonlinear, rapid, catastrophic heating regime. Subsequent to a small lattice temperature jump, determined by the ratio of the internal to lattice frequencies, lattice heating occurred in both regimes due to the exchange of vibrational energy between excited oscillators and not simply to nearest neighbors, as is assumed when using artificial molecules. In the linear regime the anharmonicity of the potential and the excitation density were used to parametrize the heating rate. The catastrophic heating regime is observed to follow a loss of order (melting) in the sample so that the excited molecules interact more efficiently. This process is much more important than the increasing $\mathrm{V}-\mathrm{T}$ energy transfer rate with increasing lattice $T$ suggested by most authors. The heating rate observed 
in this regime is similar to the vibrational cooling rate seen in the gas phase when scaled to the high internal pressures. Melting has been suggested also to be important in protonation of the analyte in MALDI. ${ }^{32}$ Therefore, melting should now be looked for in studies of materials that are better analogs to the MALDI matrix material and melting may affect the surface regions of the nonablated material. Because the disorder introduced by melting plays an important role in vibrational to translational heating, it was found that impurities also could enhance the heating and longer pulses could be more efficient, as they introduce disorder prior to producing later excitations.

Finally we showed that when there is an open surface, as in LID or MALDI, then, not surprisingly, volume expansion and sublimation cooling compete with lattice heating. At the lower excitation densities studied here, the catastrophic heating regime is not achieved in the open sample, so that desorption resembles sublimation. In this process, large impurity molecules (e.g., analytes) imbedded in the sample were found to diffuse to the surface, where they may or may not desorb. In a large sample this could result in segregation and affect analyte signals in MALDI. For higher excitation densities the resulting pressure pulse produces a rapid expansion and an ablated volume. The time scales of interest are of the order of $100 \mathrm{ps}$. Therefore, in MALDI and LID, a subthreshold regime due to sublimation is followed by an ablation regime with increasing excitation density. The ejections in these regimes are found to have distinct characteristics. The angular distributions differ, with material being composed largely of clusters and being forward directed in the early stages of ablation. The "hot" ejected clusters cool by emission of molecules so that MALDI might be called a "nanospray" process, possibly having similarities with electrospray.

Acknowledgment. The discussions with B. Chait and R. Beavis are gratefully acknowledged. This work is supported in part by the NSF Astronomy Division, NSF Chemistry Division (grant no NSF-CHE-9523413), the NSF International Programs Division, and the Polish Committee for Scientific Research under grants no. 2 P03B 13111 and KBN/SPP/UJ/034/1994. The calculations were partly performed with use of the SGI Power Onyx computer from the Institute of Physics, Jagiellonian University, and with the Convex SPP Exemplar supercomputer from the Academic Computer Center, Cracow.

\section{References and Notes}

(1) Hillenkamp, F.; Karas, M.; Beavis, R.; Chait, B. Anal. Chem. 1991, $63,119,3 \mathrm{~A}$.

(2) Karas, M.; Hillenkamp, F. Anal. Chem. 1988, 60, 229.

(3) Overberg, A.; Karas, M.; Bahr, U.; Kaufmann, R.; Hillenkamp, F. Rapid Commun. Mass Spectrom. 1990, 4, 293.

(4) Sutton, C.; Wheeler, C.; Sally, U.; Corbett, J.; Cotrell, J. Electrophhoresis 1997, 13, 424.

(5) Vertes, A. In Methods and Mechanisms For Producing Ions from Large Molecules; Standing, K., Ens, W., Eds.; Plenum Press: New York, 1996; p 275.

(6) Vertes, A.; Irinyi, G.; Gijbels, R. Anal. Chem. 1993, 65, 2389.

(7) Vertes, A.; Gijbels, R.; Levine, R. D. Rapid Commun. Mass. Spectrom. 1990, 4, 228.

(8) Karas, M.; Bahr, U.; Stahl-Zeng, J.-R. In Large Ions: Their Vaporization, Detection and Analysis; Baer, T., Ng, C., Powls, I., Eds.; John Wiley: New York, 1996.

(9) Johnson, R. In Large Ions: Their Vaporization, Detection and Analysis; Baer, T., Ng, C., Powls, I., Eds.; John Wiley: New York, 1996; p 49.

(10) Garrison, B. J.; Srivanson, S. Appl. Phys. Lett. 1984, 44, 849.

(11) Shiea, J. S. J. In Methods and Mechanisms For Producing Ions from Large Molecules; Standing, K., Ens, W., Eds.; Plenum Press: New York, 1991; p 275.

(12) Zhigilei, L.; Garrison, B. J. Phys. Chem. B 1997, 101, 2028.

(13) Zhigilei, L. V.; Kodali, P.; Garrison, B. J. Phys. Chem. B 1997, 101,2028

(14) Bencsura, A.; Vertes, A. Chem. Phys. Lett. 1995, 247, 142.

(15) Bencsura, A.; Navale, V.; Sadeghi, M.; Vertes, A. Rapid Commun. Mass. Spectrom. 1997, 11, 679.

(16) Wu, X.; Sadeghi, M.; Vertes, A. J. Phys. Chem. B 1998, 102, 4770.

(17) Holian, B. J. Chem. Phys. 1986, 84, 3138.

(18) Fain, B.; Lin, S. J. Chem. Phys. 1989, 91, 2726.

(19) Dutkiewicz, Ł.; Johnson, R.; Pędrys, R. J. Low Temp. Phys. 1998, $111,747$.

(20) Banerjee, S.; Liu, M.; Johnson, R. Surf. Sci. Lett. 1991, 255, L504.

(21) Banerjee, S.; Johnson, R.; Cui, S.-T. Phys. Rev. B 1991, 43, 12707. 32 .

(22) Dutkiewicz, Ł.; Pẹdrys, R.; Schou, J. Europhys. Lett. 1994, 27,

(23) Dutkiewicz, Ł.; Pędrys, R.; Schou, J. Phys. Rev. Lett. 1995, 75, 1407.

(24) Allen, M. P.; Tildesley, D. Computer Simulation of Liquids; Oxford University Press: Oxford, U.K., 1986.

(25) Tuckerman, M.; Berne, B.; Rossi, A. J. Chem. Phys. 1991, 94, 1465

(26) Klein, M.; Levesque, D.; Weis, J. Phys. Rev. B 1980, 21, 5785.

(27) Fenyo, D.; Johnson, R. Phys. Rev. B 1992, 46, 5090.

(28) Brooks, B.; Bruccoleri, R.; Olafson, B.; States, D.; Karplus, M. J. Comput. Chem. 1983, 4, 187.

(29) Levine, R.; Bernstein, R. Molecular Reaction Dynamics and Chemical Reactivity; Oxford University Press: Oxford, U.K., 1987.

(30) Vertes, A.; Levine, R. Chem. Phys. Lett. 1990, 171, 28.

(31) Millikan, R.; White, D. J. Chem. Phys. 1963, 39, 3209.

(32) Chen, X.; Carroll, J.; Beavis, R. J. Am. Soc. Mass Spectrom. 1998, 9,885 . 